\title{
Neurogenin 3 is important but not essential for pancreatic islet development in humans
}

\author{
Oscar Rubio-Cabezas • Ethel Codner • \\ Sarah E. Flanagan • José L. Gómez • Sian Ellard • \\ Andrew T. Hattersley
}

Received: 2 May 2014 / Accepted: 22 July 2014 / Published online: 14 August 2014

(C) The Author(s) 2014. This article is published with open access at Springerlink.com

Keywords Diabetes · Enteric anendocrinosis · Islet development $\cdot$ Neonatal diabetes $\cdot$ Neurogenin 3

\author{
Abbreviations \\ bHLH Basic helix-loop-helix \\ NEUROG3 Neurogenin 3
}

To the Editor: Based on the findings that Neurog3-null mice fail to develop pancreatic islets [1] and lack enteroendocrine cells [2], this basic helix-loop-helix (bHLH) transcription factor is considered to be essential for differentiation of endocrine cells both in the pancreas and in the gut [3]. Limited data from human fetuses suggest that transient expression of NEUROG3 in pancreatic progenitor cells from approximately 7 to 8 weeks post conception is followed by beta cell differentiation and immature islet formation by week 12 of fetal development $[4,5]$.

Homozygous loss-of-function mutations in NEUROG3 were first identified in patients with a rare form of congenital

O. Rubio-Cabezas $(\bowtie)$

Department of Pediatric Endocrinology, Hospital Infantil Universitario Niño Jesús, Instituto de Investigación Sanitaria La Princesa, Avda. Menéndez Pelayo 65, 28007 Madrid, Spain

e-mail: oscar.rubio@salud.madrid.org

E. Codner

Institute of Maternal and Child Research, University of Chile, Santiago, Chile

\section{S. E. Flanagan $\cdot$ S. Ellard $\cdot$ A. T. Hattersley}

Institute for Biomedical and Clinical Science,

University of Exeter Medical School, Exeter, UK

J. L. Gómez

Department of Pediatrics, Complejo Hospitalario Torrecárdenas, Almería, Spain malabsorptive diarrhoea that was due to enteric anendocrinosis [6]. Unexpectedly, the patients did not present with neonatal diabetes, suggesting that pancreatic islet development was at least partially spared, although they developed diabetes during late childhood. The authors hypothesised that an unidentified factor might compensate for the absence of functional neurogenin 3 (NEUROG3) and result in the production of some functional beta cells. Further studies showed that the reported mutations were hypomorphic rather than null mutations. Therefore, differences in NEUROG3 gene dosage requirements could explain the observed differential effect on the intestinal and pancreatic phenotypes [7].

We recently identified biallelic null NEUROG3 mutations in a patient with enteric anendocrinosis and permanent neonatal diabetes [8]. Although pancreatic tissue was not available for direct analysis, the presence of detectable blood C-peptide indicated that there was some endogenous insulin production at 4.5 years of age. Similar findings were later reported in a different patient [9]. Consequently, we hypothesised that, despite the pivotal role of Neurog3 in pancreatic endocrine development in mice, complete NEUROG3 deficiency does not necessarily imply an absolute insulin deficiency in humans and, therefore, affected patients may present with diabetes at any age.

To test this hypothesis, the single coding exon of NEUROG3 was amplified and sequenced from genomic DNA as previously described [8] in a further three probands with congenital malabsorptive diarrhoea and diabetes, regardless of the age at diagnosis of the latter. This study was conducted in accordance with the Declaration of Helsinki and approved by the local ethics committee. Written informed consent was obtained from the parents/guardians of the patients. Previously described homozygous missense mutations were identified in the three cases and a non-diabetic sibling of proband 3. Clinically unaffected siblings of probands 1 and 2 (one each) were unavailable for evaluation. Genetic findings 


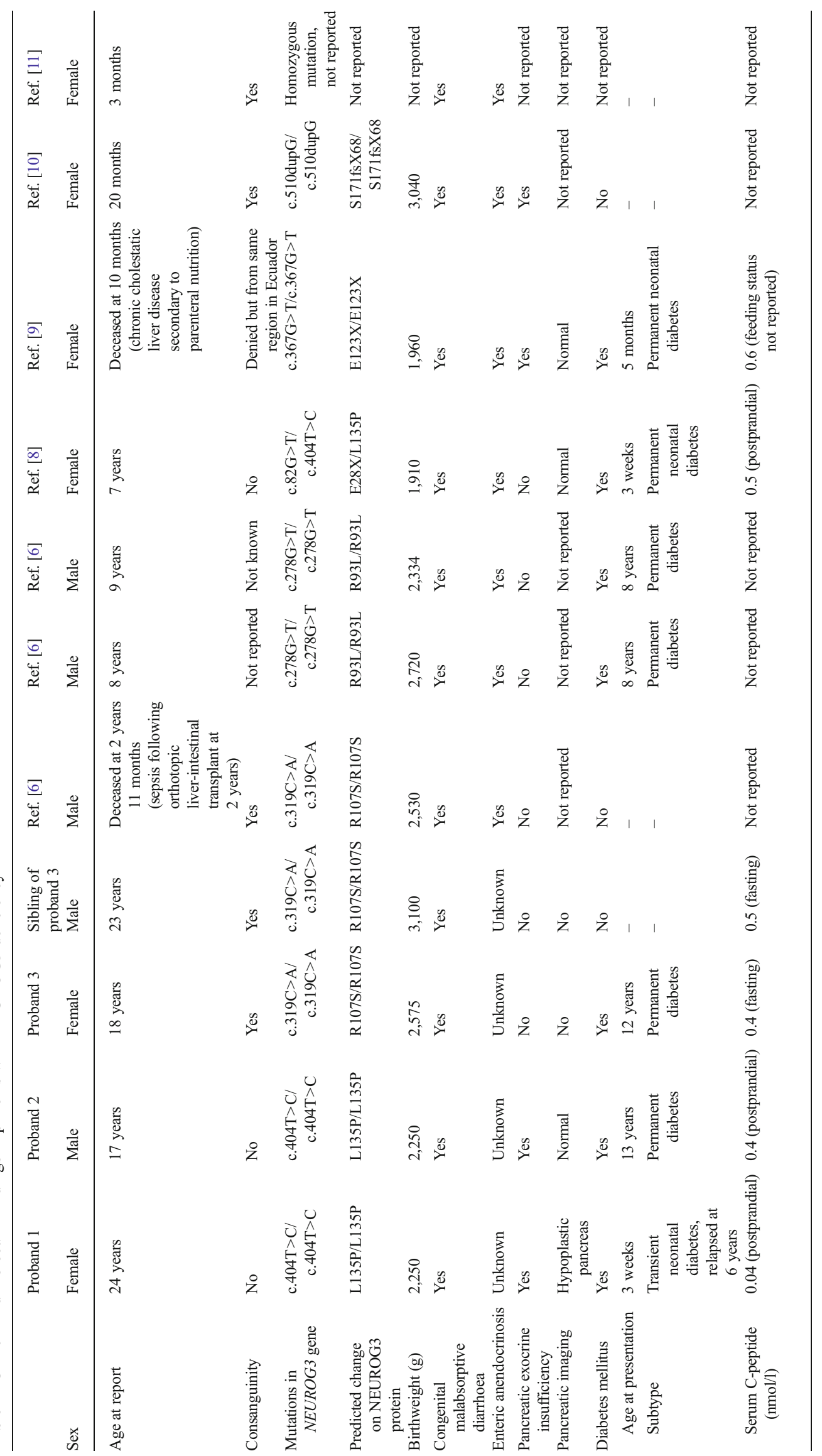


and the most significant clinical features of these patients are depicted in Table 1, along with similar data from all previous case reports on children with biallelic NEUROG3 mutations for comparison [6, 8-11]. Early-onset severe malabsorptive diarrhoea was a common finding in the 11 patients, with enteric anendocrinosis demonstrated in all cases where this condition was investigated on intestinal biopsies. Absence of enteroendocrine cells was not specifically investigated in any of the four patients included in this study, but it has previously been reported in patients with the same NEUROG3 mutations $[6,8]$. Typically, the intestinal failure requires long-term parenteral nutrition initially, but oral feedings are increasingly tolerated and parenteral nutrition can eventually be discontinued. Low fecal elastase or trypsin levels have been reported in some but not all cases, suggesting that pancreatic exocrine insufficiency represents a functional defect secondary to a lack of cholecystokinin and secretin, the enteric hormones that stimulate pancreatic exocrine secretion [10].

No evident genotype-phenotype correlation exists between NEUROG3 mutations and pancreatic endocrine function. NEUROG3 deficiency can lead to a number of different phenotypes of diabetes, including permanent neonatal diabetes, relapsing transient neonatal diabetes and childhood-onset permanent diabetes. In either case, insulin production is partially spared, as indicated by detectable, although relatively low, C-peptide levels. Furthermore, none of the diabetic patients has ever presented with severe hyperglycaemia or ketosis, not even during acute intercurrent illness, which also supports the persistence of some residual endogenous insulin production. This relative insulin deficiency might be due in part to a lack of incretin hormones (glucagon-like peptide 1, glucose-dependent insulinotropic peptide) from the gut, a hypothesis that has not been tested in NEUROG3-deficient patients so far. However, diabetes does not universally present during childhood or young adulthood, as evidenced by the non-diabetic 23-year-old male patient, although it might develop later on. In addition, the same genotype can cause different diabetic phenotypes both between and within kindreds. Overall, the data available suggest that either genetic or non-genetic modifiers are likely to play a role in the penetrance and expressivity of diabetes in NEUROG3 biallelic mutation carriers.

The presence of some residual endogenous insulin secretion in patients with NEUROG3 deficiency suggests that, in contrast to mice [3], a redundant NEUROG3-independent pancreatic endocrine developmental pathway might exist in humans so that NEUROG3 is not absolutely required for differentiation of pancreatic progenitors into islet cell precursors. In this sense, it has recently been reported that ASCL1B, another bHLH transcriptor factor, initiates the endocrine cell differentiation programme instead of NEUROG3 in zebrafish [12]. Further research in this area will help clarify the exact role of NEUROG3 in human pancreatic development.
In summary, NEUROG3 deficiency produces a rare clinical syndrome characterised by severe malabsorptive diarrhoea from early life and mild diabetes with a variable age of onset. This finding suggests that NEUROG3 is important but not essential for beta cell development and function in humans.

Acknowledgements We are grateful to A. Damhuis for technical assistance (Department of Molecular Genetics, Royal Devon \& Exeter NHS Foundation Trust, Exeter, UK). Some of the data were presented as an abstract at the 38th Annual Meeting of the International Society for Pediatric and Adolescent Diabetes (ISPAD) in 2012.

Funding ORC is funded by a Juan Rodés Clinical Research Fellowship (JR13/00018) from the Instituto de Salud Carlos III, Spain. SE and ATH are employed as core members of staff within the National Institute for Health Research funded Exeter Clinical Research Facility.

This study received funding from the Instituto de Salud Carlos III, Spain (CP11/00263) and the European Community's Seventh Framework Programme (FP7/2008-2012) under grant agreement No. 223211 (Collaborative European Effort to Develop Diabetes Diagnostics, CEED3), BOLD (EU FP7-PEOPLE-ITN-2008 Biology of Liver and Pancreatic Development and Disease), Diabetes UK and a Wellcome Trust Senior Investigator Award to SE and ATH.

Duality of interest The authors declare that there is no duality of interest associated with this manuscript.

Contribution statement ORC, SEF, SE, and ATH designed the study. ORC, EC, SEF, and JLG acquired and/or analysed the data. ORC wrote the manuscript. All authors reviewed and revised the manuscript critically. All authors approved the final version of the manuscript. ATH is the guarantor of this work and, as such, had full access to all of the study data and takes full responsibility for the integrity of the data.

Open Access This article is distributed under the terms of the Creative Commons Attribution License which permits any use, distribution, and reproduction in any medium, provided the original author(s) and the source are credited.

\section{References}

1. Gradwohl G, Dierich A, LeMeur M, Guillemot F (2000) neurogenin 3 is required for the development of the four endocrine cell lineages of the pancreas. Proc Natl Acad Sci U S A 97:1607-1611

2. Jenny M, Uhl C, Roche C et al (2002) Neurogenin3 is differentially required for endocrine cell fate specification in the intestinal and gastric epithelium. EMBO J 21:6338-6347

3. Rukstalis JM, Habener JF (2009) Neurogenin3: a master regulator of pancreatic islet differentiation and regeneration. Islets $1: 177-184$

4. Lyttle BM, Li J, Krishnamurthy M et al (2008) Transcription factor expression in the developing human fetal endocrine pancreas. Diabetologia 51:1169-1180

5. Jennings RE, Berry AA, Kirkwood-Wilson R et al (2013) Development of the human pancreas from foregut to endocrine commitment. Diabetes 62:3514-3522

6. Wang J, Cortina G, Wu SV et al (2006) Mutant neurogenin-3 in congenital malabsorptive diarrhea. N Engl J Med 355:270 280 
7. Jensen JN, Rosenberg LC, Hecksher-Sørensen J, Serup P (2007) Mutant neurogenin-3 in congenital malabsorptive diarrhea. N Engl J Med 356:1781-1782

8. Rubio-Cabezas O, Jensen JN, Hodgson MI et al (2011) Permanent neonatal diabetes and enteric anendocrinosis associated with biallelic mutations in NEUROG3. Diabetes 60:1349-1353

9. Pinney SE, Oliver-Krasinski J, Ernst L et al (2011) Neonatal diabetes and congenital malabsorptive diarrhea attributable to a novel mutation in the human neurogenin-3 gene coding sequence. J Clin Endocrinol Metab 96:1960-1965
10. Sayar E, Islek A, Yilmaz A, Akcam M, Flanagan SE, Artan R (2013) Extremely rare cause of congenital diarrhea: enteric anendocrinosis. Pediatr Int 55:661-663

11. Ohsie S, Gerney G, Gui D, Kahana D, Martín MG, Cortina G (2009) A paucity of colonic enteroendocrine and/or enterochromaffin cells characterizes a subset of patients with chronic unexplained diarrhea/ malabsorption. Hum Pathol 40:1006-1014

12. Flasse LC, Pirson JL, Stern DG et al (2013) Ascllb and Neurod1, instead of Neurog3, control pancreatic endocrine cell fate in zebrafish. BMC Biol 11:78 